\title{
GLUCOCORTICOSTEROID DURING PATCH APPLICATION TO IMPROVE SCAR OUTCOME AFTER CENTRAL VENOUS CATHETER. A RANDOMIZED TRIAL
}

\author{
Ida Bo Nissen ${ }^{1}$, Mette Møller Handrup ${ }^{1}$, Karen Bang ${ }^{2}$, and Henrik Hasle ${ }^{3}$ \\ ${ }^{1}$ Aarhus Universitetshospital Skejby \\ ${ }^{2}$ Aarhus University Hospital Skejby \\ ${ }^{3}$ Aarhus University Hospital
}

January 28, 2021

\begin{abstract}
Introduction: Children treated for cancer with a central venous catheter are often bothered by scarring. We studied whether glucocorticosteroid during patch occlusion has a beneficial effect on scar outcome in children and adolescents treated for neoplastic disease. Methods: A double-blinded placebo-controlled randomized clinical trial was performed. The main outcome was the Vancouver Scar Scale (VSS). Secondary outcomes were scar width and scar quality measured using the Patient and observer scar assessment (POSAS). The patients were divided into an intervention groups and a control group. The intervention group was randomized into two groups; Active and placebo group. The active treatment consisted of cream with glucocorticosteroid and fusidic acid. The placebo treatment consisted of cream with fusidic acid. Both groups were also treated with a silicone gel patch for three months after central venous catheter removal. The control group did not receive any specific skin care. Results: Assessment at 6 months were completed for 44 in the intervention group ( 21 in the active and 23 in the placebo group) and 47 in the comtrol group. The intervention group had a significantly lower VSS and a smaller scar compared to the control group $(\mathrm{p}=0.00, \mathrm{p}=0.02)$. The POSAS by the patients and the study nurse showed no significant difference between the intervention and control groups $(\mathrm{p}=0.84, \mathrm{p}=0.36)$. Conclusions: Silicone gel sheet alone or in combination with application of glucocorticosteroid during sheet occlusion improved scar outcome after removal of central venous catheter in children treated for neoplastic diseases.
\end{abstract}

GLUCOCORTICOSTEROID DURING PATCH APPLICATION TO IMPROVE SCAR OUTCOME AFTER CENTRAL VENOUS CATHETER. A RANDOMIZED TRIAL.

Ida Bo Nissen, $\mathrm{MD}^{1}$, Mette Møller Handrup, $\mathrm{MD}^{1}$, Karen Bang, $\mathrm{MD}^{2}$, Henrik Hasle, $\mathrm{MD}^{1^{*}}$

${ }^{1}$ Department of Pediatric and Adolescent Medicine, Aarhus University Hospital, Denmark.

${ }^{2}$ Clinical of Dermatology, Guldsmedegade 1, Aarhus, Denmark

*Correspondence to: Henrik Hasle, MD, Department of Pediatric and Adolescent Medicine, Aarhus University Hospital, Palle Juul-Jensens Boulevard 103, 8200 Aarhus N, Denmark, Tel: +45 784514 26, Email:hasle@dadlnet.dk

Word count abstract: 250

Word count main text: 2,639

No. of tables: 4 No. of figures: 2

Abbreviations 


\begin{tabular}{ll}
\hline CVC & Central Venous Catheter \\
VSS & Vancouver Scar Scale \\
mVSS & Modified Vancouver Scar Scale \\
POSAS & The patient and observer scar assessment \\
PAC & Port-a-cath \\
\hline
\end{tabular}

ABSTRACT:Introduction: Children treated for cancer with a central venous catheter are often bothered by scarring. We studied whether glucocorticosteroid during patch occlusion has a beneficial effect on scar outcome in children and adolescents treated for neoplastic disease.

Methods: A double-blinded placebo-controlled randomized clinical trial was performed. The main outcome was the Vancouver Scar Scale (VSS). Secondary outcomes were scar width and scar quality measured using the Patient and observer scar assessment (POSAS). The patients were divided into an intervention groups and a control group. The intervention group was randomized into two groups; Active and placebo group. The active treatment consisted of cream with glucocorticosteroid and fusidic acid. The placebo treatment consisted of cream with fusidic acid. Both groups were also treated with a silicone gel patch for three months after central venous catheter removal. The control group did not receive any specific skin care.

Results: Assessment at 6 months were completed for 44 in the intervention group (21 in the active and 23 in the placebo group) and 47 in the comtrol group. The intervention group had a significantly lower VSS and a smaller scar compared to the control group $(\mathrm{p}=0.00, \mathrm{p}=0.02)$. The POSAS by the patients and the study nurse showed no significant difference between the intervention and control groups $(\mathrm{p}=0.84, \mathrm{p}=$ $0.36)$.

Conclusions: Silicone gel sheet alone or in combination with application of glucocorticosteroid during sheet occlusion improved scar outcome after removal of central venous catheter in children treated for neoplastic diseases.

\section{Keywords}

Cancer, late effects, children, scar, central venous catheter

\section{Introduction}

Children and adolescents treated with chemotherapy for cancer usually need a central venous catheter (CVC) for medicine administration and blood samples. The CVC is either placed in a subcutaneous tunnel with external tubing or as a capsule located subcutaneously (port-a-cath/PAC) on the chest. After removal of the CVC the children might develop a hypertrophic scar or keloid formation on the skin. This tendency is especially increased in the skin on the front of the thorax [1], where the CVC often is located. A hypertrophic scar appears thick, hard and red, but will in most cases fade away over several years. A keloid formation may occur as a monstrously scar tissue that is not limited by the normal level of the skin. The healing process can be disrupted in one of the healing steps, e.g. at an extended inflammatory phase or disorder in the proliferative and/or remodelling phase. This results in a hypertrophic scar or keloid formation. Keloid can continue to grow in size and spread to the surrounding tissue [2]. This will be clinically evidenced by itching, pain, hypersensitivity when touched and an increased content of vessels [2]. The formation of hypertrophic scars and keloid occurs in 5-15\% of all wounds.

A large proportion of long-term survivors, who were previously treated for childhood cancer including insertion of a CVC, are bothered by their scars [3]. They describe itching and irritation and feel embarrassed due to the scars surrounding their collarbone and chest. This causes some patients to cover the scars and to avoid low-cut clothes [4]. Hypertrophic scars and keloid formation are difficult to treat once formed. Injections with intralesional steroid injections preparation with subsequent laser treatment of flushing, provides only moderately satisfactory cosmetic results [2]. 
It is common practice among dermatologists to prophylactically administer glucocorticosteroid with or without occlusion to exploit the anti-inflammatory properties of the glucocorticosteroid hormone on wound healing and thus try to avoid the hypertrophic scarring [5]. However, no randomized studies support this theory. A randomized controlled trial has been performed to test the effects of use of silicone gel sheets on children with cancer after removal of PAC. No strong evidence was provided by the study to support the use of silicone gel sheets after removal of PAC in children with cancer [6]. However, some clinical control studies have reported evidence that silicone gel is effective in preventing and reducing hypertrophic scarring [7].

The aim of this study was to test if glucocorticosteroid during patch occlusion has a beneficial effect on scar outcome, and can prevent the formation of hypertrophic scar / keloid in children and adolescents after removal of a CVC.

\section{Materials and Method}

\section{Participants}

The study was conducted at the division of pediatric oncology, department of pediatrics and adolescent medicine, Aarhus University Hospital. From January 2010 to July 2011. All children aged 0 to 17 years, who were treated for any type of neoplastic diseases and had a planned removal of a CVC due to treatment termination, were eligible. Excluding criteria were known allergy to band aid or fusidic acid.

All parents and children received oral and written study information. Both custody authorities provided a written consent. The clinical trial nr. NCT01113125 was approved by the Regional Committee on Health Research Ethics, and performed according to the Declaration of Helsinki.

\section{Design}

The study was a double-blinded placebo-controlled randomized clinical trial.

A total of 68 patients were enrolled in the intervention group and 47 patients in the control group. Randomization was performed one week before removal of the CVC. The randomization was performed by the pharmacy at Aarhus University Hospital who also wrapped and labelled the cream. In the intervention group 34 patients were assigned to fusidic acid with betamethasone during patch occlusion (active group), and 34 were assigned to fusidic acid during patch occlusion (placebo group). Of the 68 patients assessed for eligibility and randomization, 24 participants were excluded due to lack of acceptance of the patch by the child, forgetting to lubricate with the cream or relapse of the malignancy. The 47 patients in the control group were retrospectively collected among patients at the division of pediatric oncology, department of pediatrics, Aarhus University Hospital, who had their CVC removed in the years immediately before the start of the trial (Fig. 1).

\section{Hosted file}

image1.emf available at https://authorea.com/users/392288/articles/506241glucocorticosteroid-during-patch-application-to-improve-scar-outcome-after-centralvenous-catheter-a-randomized-trial

\section{Hosted file}

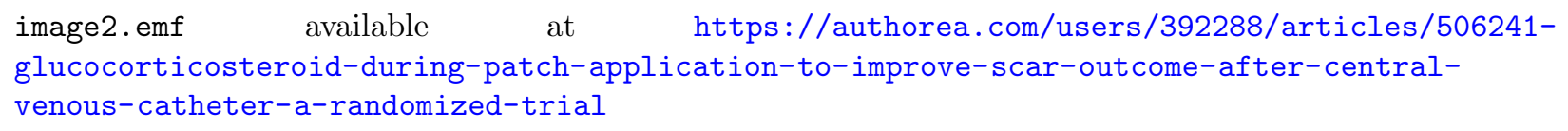

Figure 1. Flow chart of the study recruitment and follow-up process. n, number of patients.

\section{Intervention}

All patients in the intervention groups received cream containing fusidic acid to prevent staphylococcal infection. The active group received glucocorticosteroid and fusidic acid cream, while the placebo group received fusidic acid cream. The cream had identical appearance. Both groups had $0.4 \mathrm{~mm}$ active or placebo 
cream applied to the insertion site under occlusion with patch one week before and three weeks after removal of the CVC. Patch and cream treatment was applied every three days or sooner if needed, e.g. if the patch came off. After three weeks, both groups covered their scar with a silicone gel sheet patch for three months, to avoid pull on the scar. The cream tube was returned after completion of the study period and a control weight was performed by the study nurse thereby controlling the use of cream. The parents were also asked whether they had forgotten to apply the cream at any time. The control group did not receive any kind of skin care after removal of the CVC.

\section{Outcome measures}

All participants had their scar assessed after three weeks, six months and twelve months after removal of the CVC. The assessment was conducted by a study nurse and a dermatologist. The dermatologist had fewer observations than the study nurse. The study nurse assessed the scar by using the Vancouver Scar Scale (VSS) [8] and the Patient and Observer Scar Assessment Scale (POSAS) version 1 [9] at each clinical check every three weeks, six months and twelve months, after removal of the CVC. The parents and patients assessed the POSAS three weeks, six months and twelve months at the clinical check. Digital clinical images of the scar were taken after three weeks, six months and twelve months after removal of the CVC by a professional clinical photographer affiliated with Aarhus University Hospital. In all pictures, a ruler was applied to the skin, allowing the scar width to be standardized. The clinical images were provided blinded to the dermatologist for assessment of VSS and scar width. In the present study, the dermatologist categorized the interventions groups' skin type according to Fitzpatrick skin type classification scale [10]. Patients with a Fitzpatrick 3 or higher skin type are more likely to develop keloid scar formation [11]. However, this is not clear for hypertrophy [12].

The VSS assesses four variables: Pigmentation, pliability, height and vascularity. It has a total score of 14, where 0 is equivalent to the patients' normal skin [9]. The POSAS consists of two scales: One completed by the patient and one by the observer. The patients' scale contains six questions with regards to relief, pain, color, pliability, thickness and itching. The observer scale involves five questions regarding to pliability, thickness, relief, pigmentation and vascularity. Each parameter uses a 10-point scoring system, with 1 representing normal skin and 10 corresponding to the worst imaginable scar. The total score of the patient scale consists of adding the scores of each of the six items from 6 to 60 . The observer scale consists of five items and therefore, the total score ranges from 5 to 50 . At total score of 6 and 5 , respectively, reflects normal skin [9]. The main outcome was the Vancouver Scar score. Secondary outcomes were scar width and scar quality measured using the POSAS.

Statistical analysis

Data were analysed using SPSS version 26 (IBM Corporation, Armonk, New York). Univariate differences between groups were assessed using A Mann-Whitney test. This test method was selected because the data was not normally distributed. Median and standard deviations were used to describe the data. The MannWhitney test was used for comparing the values for VSS, POSAS and scar width between the groups. A P value [?] 0.05 was considered statistically significant.

\section{Results}

Of the 68 patients allocated to the randomization, 34 were assigned to a placebo group. A total of 24 patients were lost at the 6-month follow-up and 8 additional patients were lost at the 12-month follow-up. In the control group 49 patients were enrolled, two patients were lost to follow-up (Fig. 1). All children were diagnosed with neoplastic diseases and had finished their cancer therapy at the time of removal of CVC. The patient demographics are summarized in Table I. No significant differences were found in the patient demographics between the groups.

Only two patients had non-Caucasian skin type; one patient with skin type 2 and one with skin type 5 . The rest had skin type 1 . The categorization was not made for the control group. Therefore, the skin type is not considered in the present study. 


\section{Vancouver Scar Score}

Table II depicts the VSS assessed by the study nurse and the dermatologist. The data assed by the study nurse show that the intervention group and the control group had the same VSS score after 12 months $(\mathrm{p}=$ $0.86)$.
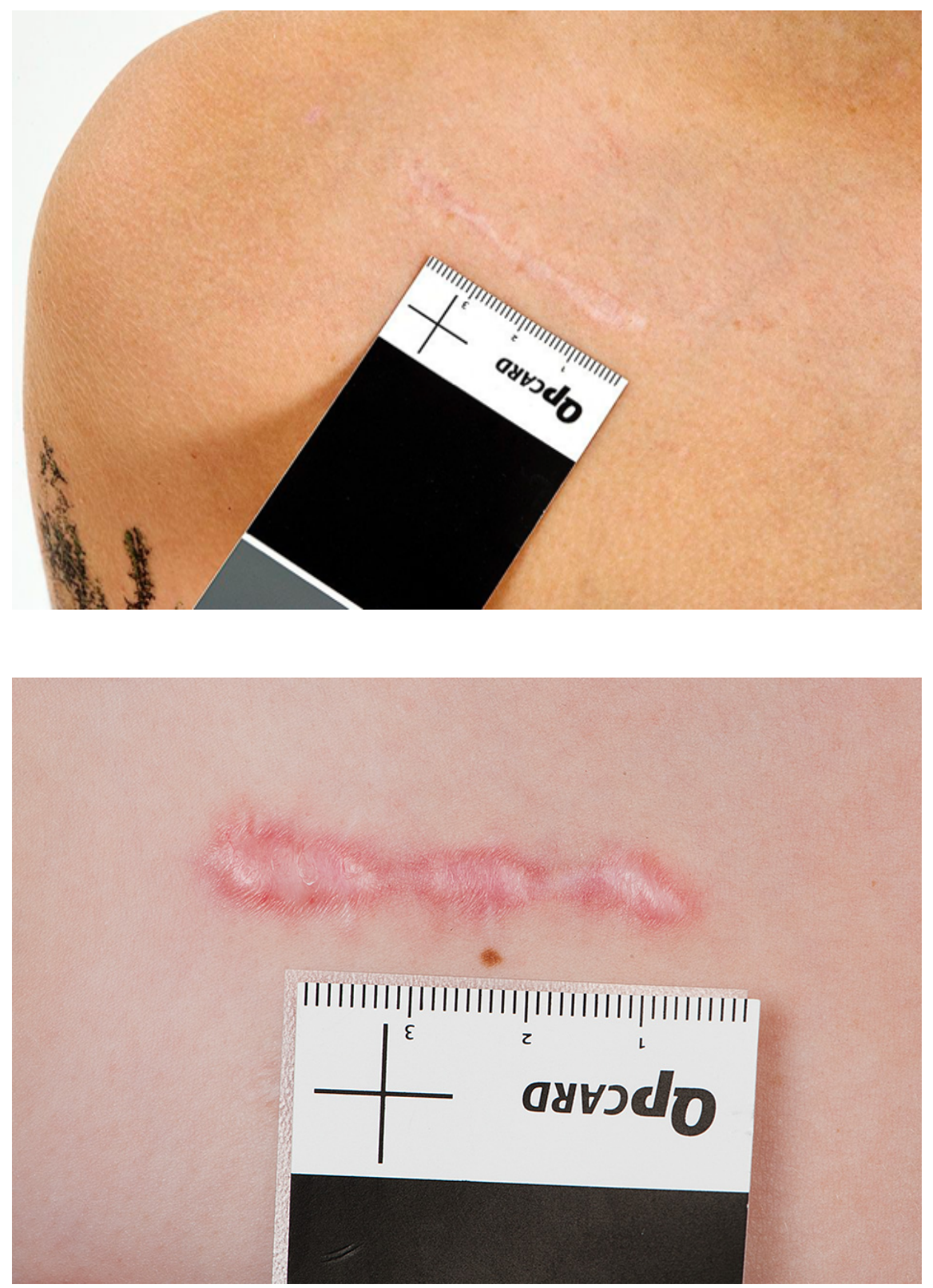

Figure 2A and 2B. Examples of scar 6 months after removal of the CVC. Figure 2A is a scar with VSS score 2. Figure $2 \mathrm{~B}$ is a scar with VSS score 12 .

The VSS assessed by the dermatologist showed a significant lower score in the intervention group (active and placebo) compared with the control group at 12 months $(\mathrm{p}=0.0001)$. Based on the digital photos, the dermatologist found four patients with a hypertrophic scar among the intervention group, distributed on 
two in the active group and two in the placebo group. Figure 2A and $\mathrm{B}$ illustrate examples of the digital photos of the scar 6 months after removal of the CVC.

\title{
Hosted file
}

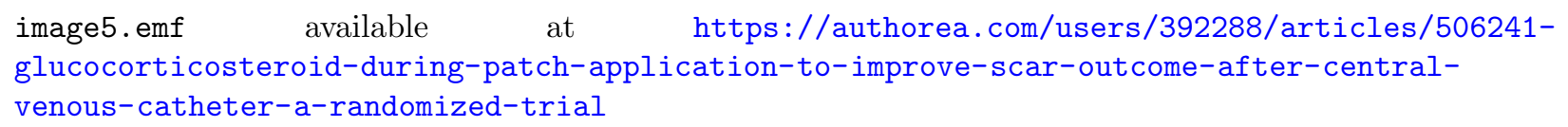

\section{Hosted file}

image6.emf available at https://authorea.com/users/392288/articles/506241glucocorticosteroid-during-patch-application-to-improve-scar-outcome-after-centralvenous-catheter-a-randomized-trial

Scar width and scar quality

Table III shows that the intervention group had a lower median of scar width at 12 months than the control group which was a significant difference $(\mathrm{p}=0.02)$.

The POSAS was used to measure scar quality. Unlike VSS, the POSAS has an extra dimension because the patient's opinion is mandatory for a complete scar assessment. Table IV shows POSAS assessed by the patient. The POSAS assessed by the patients showed no significant difference between the intervention and control groups $(\mathrm{p}=0.84)$. As table 4 depicts the POSAS assessed by the study nurse showed no significant difference between intervention and control group $(\mathrm{p}=0.36)$.

\section{Hosted file}

image7.emf available at https://authorea.com/users/392288/articles/506241glucocorticosteroid-during-patch-application-to-improve-scar-outcome-after-centralvenous-catheter-a-randomized-trial

\section{Hosted file}

\author{
image8.emf available at https://authorea.com/users/392288/articles/506241- \\ glucocorticosteroid-during-patch-application-to-improve-scar-outcome-after-central- \\ venous-catheter-a-randomized-trial
}

\section{Discussion}

In this study, we examined efficacy of application of glucocorticosteroid during patch occlusion and subsequently silicone gel sheet. We hypothesized that the combination of glucocorticosteroid and patch occlusion would be effective in reducing the development of hypertrophic scar/keloid after removal of CVC on children and adolescents treated for neoplastic diseases. We also expected that scar width and children's or their parents' subjective perception of the scar would be improved by using glucocorticosteroid during patch occlusion.

The results of the VSS assessed by the study nurse were different from the data from the dermatologist. The study nurse generally assessed VSS higher than the dermatologist. The VSS is developed to rate burn scars, but it also has an acceptable internal consistency for linear scars (Chronbach's alpha 0.79). The inter-observer reliability is found substantial (Intraclass coefficient correlation 0.78) [10]. However, Truong et al. do not describe whether the observer using the VSS should have dermatological experience [10]. In the present study, the study nurse was specialized in pediatric oncology as opposed to the dermatologist, who had considerable experience in assessing scars. Therefore, data from the dermatologist are considered to be the most reliable.

Data from the dermatologist showed that the intervention group had a lower VSS score than the control group 12 months after removal of the CVC. Common for the two groups in the intervention group (active and placebo) was the use of sheet occlusion. Both groups had cream applied under patch occlusion one week 
before, and three weeks after removal of the CVC. After three weeks, both groups covered their scar with a silicone gel sheet for three months, to avoid pull on the scar. Since the cream received by the two groups was different (placebo and glucocorticosteroid), the significant lower VSS score may be caused by the sheet. Future studies may be able to detect the effect more robustly by including more patients in the intervention groups.

By searching the literature, we found only two papers on CVC cars in children with cancer. Braam et al. (2015) [6] performed a three-arm randomized controlled trial. The aim of the study was to evaluate the effects of using silicone gel sheets on scar outcome after removal of PAC on children with cancer. Two intervention groups were included; One received two months of silicone gel sheets application, and one received six months of silicone gel sheet application. The control group received no additional care or treatment. No significant improvement of scar outcome was found when using silicone gel sheets after the PAC removal. A small benefit for scar width with application for two months was found [6]. In the present study, both the active and the placebo groups used a silicone gel sheet for three months and had a better outcome than the control group. Overall, the intervention group had a significantly lower VSS score than the control group $(\mathrm{p}=0.00)$. This is supported by a review investigated the effectiveness of silicone gel and silicone gel sheeting for the prevention of hypertrophic or keloid scarring on patients with newly healed wounds. However, they also found that most of the trials had poor quality with high or uncertain risk of biases in the design or the conduct [7]. Overall, there is a need for larger studies and interventions that can distinguish between the effect of silicone gel sheet and the effect of glucocorticosteroid in combination with silicone gel sheet.

The POSAS was in combination with the VSS used to assess the scars. The POSAS consists of an extra dimension because the patients' opinion is required for a complete scar evaluation. The patients' opinion does not figure in the VSS. The POSAS assessed by the patients and the study nurse showed that the intervention group had a lower score than the control group, which is consistent with the results for the VSS assessed by the dermatologist. However, the differences in the POSAS was not significant.

The results should be interpreted with caution due to the study limitations. The study included a relatively small sample size in the intervention group. A total of 69 patients were assessed for eligibility, 33 patients were excluded inter alia due to failure to follow the recommended treatment and relapse. This must be expected when working with children with cancer.

Another limitation is the fact that the 47 patients in the control group were collected retrospectively. Therefore, we cannot exclude that the parents applied a patch in the period following removal of the CVC, but it was not part of the recommendations from the department. No record was made whether children covered their scar with a patch after removal of the CVC. But the probability that they have used a patch with silicone gel seems small, both because it is not a standard patch and because it is expensive.

Furthermore, most of the participants had a PAC, which limits the conclusions that can be derived concerning tunnelled central venous catheter.

\section{Conclusion}

The results of the present study indicate that silicone gel sheet alone or in combination with application of glucocorticosteroid during sheet occlusion may improve scar outcome after removal of CVC in children treated for neoplastic diseases. Data assessed by the dermatologist showed significantly improved scar outcome for the intervention groups, however the result was not supported by the data assessed by the study nurse and the patients. The POSAS assessed by the patients and the study nurse showed no statistically significant difference between the intervention and control groups.

Overall, there is a need for larger studies and interventions that can distinguish between the effect of silicone gel sheet and the effect of glucocorticosteroid in combination with silicone gel sheet.

\section{Literature}


1. Wolfram D, Tzankov A, Pulzl P, Piza-Katzer H. Hypertrophic scars and keloids-a review of their pathophysiology, risk factors, and therapeutic management. Dermatol Surg 2009; 35(2):171-181.

2. Robles DT, Berg D. Abnormal wound healing: keloids. Clin Dermatol 2007; 25(1):26-32.

3. Braam KI, Ekkelkamp S, van Duhmen-den Boreder E, Griffloen S, Aarsman C, Schouten-van Meeteren AY. Scars due to central venous access: consequences for childhood cancer survivor. Pediatr Blood Cancer 2004; 43(4):496.

4. Calaminus G, Weispach S, Teske C, Gobel U. Quality of survival in children and adolescents after treatment for childhood cancer: the influence of reported late effects on health related quality of life. Klin Padiatr 2007; 219(3):152-157.

5. Oikarinen A, Autio P. New Aspects of the Mechanism of Corticosteroid - Induced Dermal Atrophy. Clin Exp Dermatol. 1991 Nov;16(6):416-419.

6. Braam KI, Kooijmans ECM, Broeder EVD, Veening MA, Meeteren AYNS, Verhaegen PDHM, Kaspers GJL, Niessen FB, Heij HA. No Efficacy for Silicone Gel Sheeting in Prevetion of Abnormal Scar Formation in Children with Cancer: A Randomized Controlled Trial. Plastic and Reconstructive Surgery. 2015 Apr;135(4):1086-1094.

7. Kui-Chang H, Chih-Wei L, Yi.When T. Review of Silicone Gel Sheeting and Silicone Gel for the Prevention of Hypertrophic Scars and Keloids. Wounds. 2017 May;29(5):154-158

8. Baryza MJ, Baryza GA. The Vancouver Scar Scale: an

administration tool and its interrater reliability. J Burn Care Rehabil 1995;16(5):535-538.

9. Draaijers LJ, Tempelman FR, Botman YA, Tuinebreijer WE, Middelkoop E, Kreis RW, et al. The patient and observer scar assessment scale: a reliable and feasible tool for scar

evaluation. Plast Reconstr Surg 2004;113:1960-1965.

10.Truong PT, Lee JC, Soer B, et al. Reliability and validity testing of the Patient and Observer Scar Assessment Scale in evaluating linear scars after breast cancer surgery. Plast Reconstr Surg 2007; 119(2):487494.

11. Fitzpatrick TB. The validity and practicality of sun-reactive skin type I-VI. Arch Dermatol. 1988;124:869871.

12. Shaffer JJ, Taylor SC, Cook-Bolden F. Keloidal scars: a review with a critical look at therapeutic options. J AmAcad Dermatol. 2002;46(2):S63-S97.

Figure legends

Figure 1. Flow chart of the study recruitment and follow-up process. n, number of patients.

Figure $2 \mathrm{~A}$ and 2B. Examples of scar 6 months after removal of the CVC. Figure 2A is a scar with VSS score 2. Figure 2B is a scar with VSS score 12 .

\section{Hosted file}

TABLE I.pdf available at https://authorea.com/users/392288/articles/506241glucocorticosteroid-during-patch-application-to-improve-scar-outcome-after-centralvenous-catheter-a-randomized-trial

\section{Hosted file}

TABLE II.pdf available at https://authorea.com/users/392288/articles/506241glucocorticosteroid-during-patch-application-to-improve-scar-outcome-after-centralvenous-catheter-a-randomized-trial 


\section{Hosted file}

TABLE III.pdf available at https://authorea.com/users/392288/articles/506241glucocorticosteroid-during-patch-application-to-improve-scar-outcome-after-centralvenous-catheter-a-randomized-trial

\section{Hosted file}

TABLE IV.pdf available at https://authorea.com/users/392288/articles/506241glucocorticosteroid-during-patch-application-to-improve-scar-outcome-after-centralvenous-catheter-a-randomized-trial

\section{Hosted file}

Figure 1.pdf available at https://authorea.com/users/392288/articles/506241glucocorticosteroid-during-patch-application-to-improve-scar-outcome-after-centralvenous-catheter-a-randomized-trial 

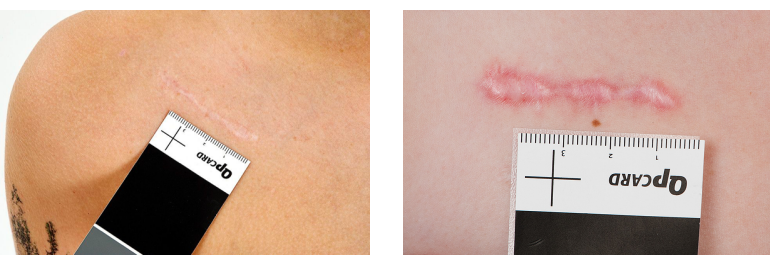